Article

\title{
Proteomic Signatures of Clostridium difficile Stressed with Metronidazole, Vancomycin, or Fidaxomicin
}

\author{
Sandra Maaß ${ }^{1, *(\mathbb{D}, \text { Andreas Otto }}{ }^{1}$, Dirk Albrecht ${ }^{2}$, Katharina Riedel ${ }^{2}$, Anke Trautwein-Schult ${ }^{1}$ \\ and Dörte Becher ${ }^{1}$ \\ 1 Institute of Microbiology, Department of Microbial Proteomics, University of Greifswald, \\ Felix-Hausdorff-Str. 8, 17489 Greifswald, Germany; andreas.otto@uni-greifswald.de (A.O.); \\ anke.trautwein-schult@uni-greifswald.de (A.T.-S.); dbecher@uni-greifswald.de (D.B.) \\ 2 Institute of Microbiology, Department of Microbial Physiology and Molecular Biology, \\ University of Greifswald, Felix-Hausdorff-Str. 8, 17489 Greifswald, Germany; \\ dirk.albrecht@uni-greifswald.de (D.A.); riedela@uni-greifswald.de (K.R.) \\ * Correspondence: sandra.maass@uni-greifswald.de; Tel.: +49-3834-420-5921
}

Received: 24 October 2018; Accepted: 13 November 2018; Published: 15 November 2018

check for updates

\begin{abstract}
The anaerobic pathogen Clostridium difficile is of growing significance for the health care system due to its increasing incidence and mortality. As $C$. difficile infection is both supported and treated by antibiotics, a deeper knowledge on how antimicrobial agents affect the physiology of this important pathogen may help to understand and prevent the development and spreading of antibiotic resistant strains. As the proteomic response of a cell to stress aims at counteracting the harmful effects of this stress, it can be expected that the pattern of a pathogen's responses to antibiotic treatment will be dependent on the antibiotic mechanism of action. Hence, every antibiotic treatment is expected to result in a specific proteomic signature characterizing its mode of action. In the study presented here, the proteomic response of $C$. difficile 630 $\mathrm{\Delta erm}$ to vancomycin, metronidazole, and fidaxomicin stress was investigated on the level of protein abundance and protein synthesis based on 2D PAGE. The quantification of 425 proteins of $C$. difficile allowed the deduction of proteomic signatures specific for each drug treatment. Indeed, these proteomic signatures indicate very specific cellular responses to each antibiotic with only little overlap of the responses. Whereas signature proteins for vancomycin stress fulfil various cellular functions, the proteomic signature of metronidazole stress is characterized by alterations of proteins involved in protein biosynthesis and protein degradation as well as in DNA replication, recombination, and repair. In contrast, proteins differentially expressed after fidaxomicin treatment can be assigned to amino acid biosynthesis, transcription, cell motility, and the cell envelope functions. Notably, the data provided by this study hint also at so far unknown antibiotic detoxification mechanisms.
\end{abstract}

Keywords: Clostridiodes difficile; antibiotics; proteomics; protein synthesis; 2D PAGE

\section{Introduction}

As a spore-forming anaerobic bacterium that primarily causes antibiotic-associated diarrhea, Clostridium difficile (recently reclassified from Peptoclostridium difficile to Clostridiodes difficile [1]) represents an enormous financial burden for the health care system [2]. This is even more important as the incidence in hospitals is increasing, both in frequency and severity, resulting in considerable morbidity and mortality. In clinical practice most patients experiencing a first episode of Clostridium-associated diarrhea are cured by treatment with metronidazole or vancomycin, the former preferred for mild or moderate episodes and the latter for severe episodes [3-5]. Both antibiotics exhibit different chemical structures resulting in varying mechanisms of action. The glycopeptide 
antibiotic vancomycin inhibits cell wall synthesis by binding to the D-Ala-D-Ala residue of the UDP-MurNAc-pentapeptide [6], an essential precursor in the peptidoglycan synthesis. For a long time, oral vancomycin was the only therapy approved to treat $C$. difficile infection (CDI), and therefore it is currently regarded as the gold standard in clinical trials. However, today vancomycin is no longer considered a first-line treatment due to the increasing incidence of vancomycin-resistant enterococci $[7,8]$, which continue to be a clinical challenge despite the availability of new therapeutic agents [9].

The nitroimidazole antibiotic metronidazole in its partially reduced form inhibits nucleic acid synthesis by disrupting the DNA of anaerobic microbial cells. However, increasing resistance of C. difficile against metronidazole has been reported [10-13]. Moreover, this drug was associated with central nervous system neurotoxicity in patients [14].

Although application of either vancomycin or metronidazole usually cures CDI, $20-25 \%$ of patients have recurrent disease after the treatment is stopped [15]. Due to its limited activity against the normal gut flora and lower relapse rates, fidaxomicin is now more frequently used for CDI treatment, especially for recurrent cases $[16,17]$. The macrocyclic antibiotic fidaxomicin (previously known as OPT-80, PAR-101, tiacumicin B, and difimicin) binds to the DNA template-RNA polymerase complex and prevents the initial separation of DNA strands, which inhibits mRNA synthesis by hindering the RNA polymerase $\sigma$-subunit [18]. Fidaxomicin received approval in Germany and in the USA in 2011 for CDI treatment in adults. Since then, it is applied more frequently, as it provides improved sustained cure rates compared to vancomycin or metronidazole $[8,19]$. Indeed, fidaxomicin demonstrates only moderate inhibitory activity against the healthy colonic flora, including Gram-positive bacteria other than $C$. difficile, anaerobes, and enteric Gram-negative bacilli [20]. This may be explained by its unique target site, which differs among bacterial species [21].

As various $C$. difficile strains are already resistant to many antibiotics, particularly in the case of quinolones and the emergent ribotype 027 strains [22], it is of increasing importance to understand the detailed adaptation mechanisms of this pathogen to antibiotic treatment. In order to do this in a comprehensive manner, it is beneficial to detect not only changes in protein amounts, but also in protein synthesis. Upon cellular stress protein synthesis rates are rapidly changing in order to react to the varying conditions. Significant differences in protein synthesis can therefore be detected with high sensitivity at an early time point after antibiotic treatment. On the other hand, the protein amount always results from the extent of protein synthesis and the extent of simultaneous protein degradation. Indeed, changes in protein abundance occur much slower compared to changes in protein synthesis and are therefore harder to observe during the early phases of adaptation. Changes in protein synthesis can be observed easily by separating radioactively pulse-labeled proteins in 2D gels. Hence this technique is still the most sensitive and most widely applied approach for the analysis of protein synthesis rates [23-25].

In this study, the response of $C$. difficile $630 \Delta$ erm to vancomycin, metronidazole, or fidaxomicin stress was investigated in the exponential growth phase. Therefore, protein synthesis rates and changes in protein amounts were quantified during the first $30 \mathrm{~min}$ after antibiotics addition. Comparative 2D PAGE-based analysis of protein patterns revealed signature proteins for each treatment, and therefore, provides new insights into antibiotic-caused physiological disturbance and potential development of antibiotic resistance.

\section{Materials and Methods}

\subsection{Bacterial Growth and Antibiotic Stress}

Clostridium difficile $630 \Delta$ erm [26] was grown at $37^{\circ} \mathrm{C}$ in brain heart infusion (BHI) in an anaerobic chamber. In the exponential growth phase $\left(\mathrm{OD}_{600 \mathrm{~nm}}\right.$ of 0.4$)$, cultures were stressed with sublethal concentrations of vancomycin $(13 \mu \mathrm{g} / \mathrm{mL})$, metronidazole $(3.75 \mu \mathrm{g} / \mathrm{mL})$, or fidaxomicin $(4 \mathrm{ng} / \mathrm{mL})$, 
respectively, resulting in reduced growth rates compared to an untreated control culture (Figure 1). Experiments were performed in three biological replicates.

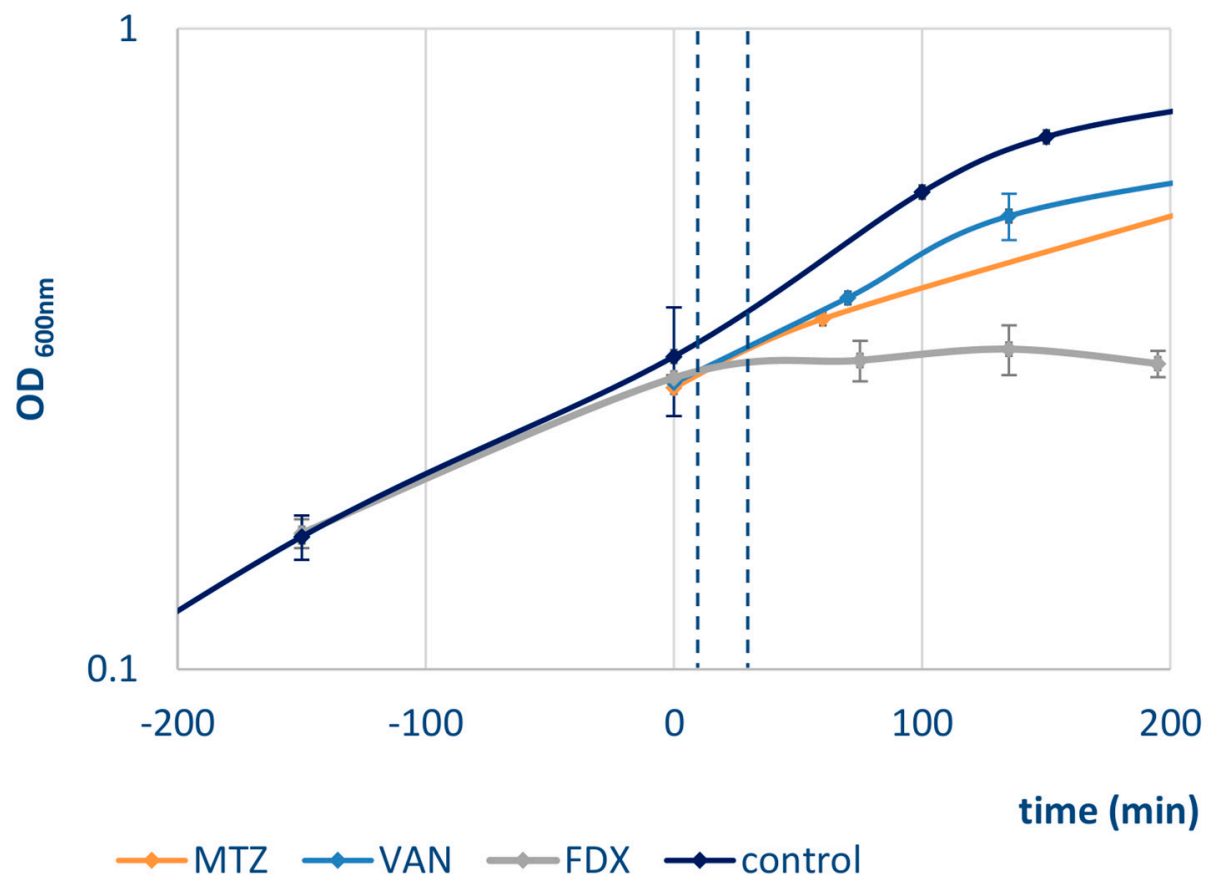

Figure 1. Growth of $C$. difficile $630 \Delta$ erm was performed at $37^{\circ} \mathrm{C}$ in brain heart infusion (BHI) in an anaerobic chamber. At time $=0 \mathrm{~min}$ (exponential growth phase, $\mathrm{OD}_{600 \mathrm{~nm}}$ of 0.4 ) cultures were stressed with either metronidazole (MTZ, orange), vanomycin (VAN, light blue) or fidaxomicin (FDX, grey) or left untreated (control, dark blue). The antibiotic concentration used resulted in reduced growth rates compared to an untreated control culture. Stress samples were taken $10 \mathrm{~min}$ and $30 \mathrm{~min}$ after addition of the antibiotic (dashed lines).

\subsection{Radioactive Pulse-Labeling, Cell Harvest, and Protein Preparation}

Cultures were pulse-labeled for 10 min each with $150 \mu \mathrm{Ci}$ of L- $\left[{ }^{35} \mathrm{~S}\right]$-methionine per $\mathrm{mL}$ at an $\mathrm{OD}_{600 \mathrm{~nm}}$ of 0.4 (for control), and $10 \mathrm{~min}$ and $30 \mathrm{~min}$ after antibiotic stress as described previously [27]. Briefly, L- $\left[{ }^{35} \mathrm{~S}\right]-$ methionine incorporation was stopped by adding unlabeled methionine $(100 \mathrm{mM})$ and chloramphenicol $(1 \mathrm{mg} / \mathrm{mL})$. Cells were harvested by centrifugation and were subsequently resuspended in TE-buffer containing the protease inhibitor PMSF $(10 \mathrm{mM}$ Tris, $5 \mathrm{mM}$ EDTA (ethylenediaminetetraacetic acid), 1 mM PMSF (phenylmethylsulfonyl fluoride), $\mathrm{pH}$ 7.5). Cells were mechanically disrupted using the Precellys 24 homogenizator (PeqLab, Erlangen, Germany; $3 \times 30 \mathrm{~s}$ at $6.5 \mathrm{~m} \mathrm{~s}^{-1}$ ). Protein concentrations of extracts were determined using the Bradford assay [28] and incorporated radioactivity was determined by scintillation counting.

\subsection{D PAGE and Autoradiography}

Isoelectric focusing was done in the pH range 4-7 with $18 \mathrm{~cm}$ IPG strips (SERVA, Heidelberg, Germany), loaded with $100 \mu \mathrm{g}$ protein in rehydration solution as described elsewhere [29]. Focusing occurred in the Protean i12 IEF Cell (Bio-Rad, München, Germany) in a five-stepped voltage gradient (150 V until $150 \mathrm{Vh}, 300 \mathrm{~V}$ until $300 \mathrm{Vh}, 600 \mathrm{~V}$ until $600 \mathrm{Vh}, 1500 \mathrm{~V}$ until $1500 \mathrm{Vh}$, and $3000 \mathrm{~V}$ until $57.5 \mathrm{kVh}$ had been reached). The SDS PAGE was done in the horizontal system HPE Tower (SERVA) using precast gels (2D HPE Large Gel NF 12.5\%, SERVA). Gels were stained with LavaPurple (SERVA) according to the manufacturer's instructions. Stained protein spots were detected with a Typhoon 9400 multi-mode imager (GE Healthcare Europe, Frankfurt, Germany) by excitation with the green laser $(532 \mathrm{~nm})$ and detection with a $560 \mathrm{~nm}$ long-pass filter. Subsequently, gels were dried 
on a chromatography paper backing by using a heated vacuum dryer. For autoradiography of the radioactively labeled protein pattern, dried gels were exposed to storage phosphor screens (Molecular Dynamics Storage Phosphor Screen, 20 by $25 \mathrm{~cm}$ ) for a time span corresponding to the amount of radioactivity separated on the gel and ensuring usage of the whole dynamic range by the strongest spot. Screens were scanned using a Typhoon 9400 multi-mode imager at a resolution of $200 \mu \mathrm{m}$ by excitation with a red laser $(633 \mathrm{~nm})$ and detection with a $390 \mathrm{~nm}$ band-pass filter.

\subsection{Processing of Gel Images and Statistical Analysis}

Gel images were analyzed employing Delta2D 4.6.3 software (Decodon GmbH, Greifswald, Germany). Normalized spot volumes were exported from the software and the z-score transformed before running an ANOVA-test for each antibiotic analyzed (control vs. $10 \mathrm{~min}$ stress vs. $30 \mathrm{~min}$ stress). Spots and proteins with significantly changed amount exhibited a $p$-value $<0.05$ and an average $\log _{2}$ fold change $>|0.8|$.

\subsection{Spot Identification Using MALDI-TOF-MS}

All spots visualized by the fluorescent stain were selected for identification with MALDI-TOF-MS according to previously published protocols [30]. Briefly, protein spots were excised from stained gels using an Ettan spot picker (Amersham Biosciences, Freiburg, Germany) and spots of similar staining intensity were sorted into 96-well microplates Greiner Bio-One (Greiner, Frickenhausen, Germany). Tryptic digest with subsequent spotting onto a MALDI target was carried out automatically with the Ettan Spot Handling Workstation (Amersham Biosciences). Gel pieces were washed, dried, and digested using trypsin. The concentration of trypsin was adjusted to the intensity of protein spots in order to ensure that the protease-to-protein concentration was in an acceptable range for proteins of differing abundances. Peptides were extracted from the gel, transferred into a new microplate, and dried completely. Dried peptides were resuspended in a matrix ( $\alpha$-cyano-4-hydroxycinnamic acid) and spotted onto the MALDI target. Samples were allowed to dry on target at room temperature before measurement. Analysis by MALDI-TOF was carried out on an AB SCIEX TOF/TOF 5800 Analyzer (AB Sciex, Darmstadt, Germany). Spectra were recorded in a mass range from 900 to 3700 Da with a focus mass of $1700 \mathrm{Da}$. For one main spectrum, 25 sub-spectra with 100 shots per sub-spectrum were accumulated using a random search pattern. If the autoproteolytic fragment of trypsin with the mono-isotopic $(\mathrm{M}+\mathrm{H})^{+} \mathrm{m} / \mathrm{z}$ at 2211.104 reached a $\mathrm{S} / \mathrm{N}$ of at least 40 , an internal calibration was automatically performed as one-point-calibration using this peak. The standard mass deviation was below $0.15 \mathrm{Da}$. Only if the automatic mode failed (in less than $1 \%$ ), calibration was carried out manually. The five most intense peaks from the MS spectra were selected for MS/MS analysis. To acquire a dependent MS/MS scan, 20 sub-spectra with 125 shots per sub-spectrum were accumulated using a random search pattern. The internal calibration was automatically performed as one-point-calibration with the mono-isotopic arginine $(\mathrm{M}+\mathrm{H})^{+} \mathrm{m} / \mathrm{z}$ at 175.119 or lysine $(\mathrm{M}+\mathrm{H})^{+} \mathrm{m} / \mathrm{z}$ at 147.107 if these peaks reached a $\mathrm{S} / \mathrm{N}$ of at least 5. Peak lists were created using GPS Explorer Software Version 3.6 (build 332) with the following settings: 900-3700 Da mass range, 20 peaks per 200 Da peak density, minimal S/N of 15 and a maximum of 65 peaks per spot. For MS/MS settings, a mass ranged from $60 \mathrm{Da}$ to reduced mass of precursor $(-20 \mathrm{Da})$, a peak density of 50 peaks per $200 \mathrm{Da}$ and maximal 65 peaks per precursor were used. Peak lists were created for a minimal S/N of 10. All peak lists were analyzed using the Mascot search engine version 2.4.1 (Matrix Science Ltd., London, UK) with a specific database for $C$. difficile 630 $\Delta$ erm [31]. Identifications were considered as correct if the protein score was $>51$. 


\section{Results}

In this study, a method for radioactive protein labeling with ${ }^{35} \mathrm{~S}$ in $\mathrm{C}$. difficile was developed. Moreover, comparative 2D PAGE was applied for the first time to detect, visualize, and quantify protein amounts and protein synthesis rates during stress adaptation in $C$. difficile. This approach was used to separate proteins synthesized 10 and $30 \mathrm{~min}$ after antibiotic stress in the laboratory strain C. difficile 630 $\Delta \mathrm{erm}$. Adaptation to antibiotics was monitored after adding sublethal concentrations of vancomycin, metronidazole, or fidaxomicin to exponentially growing cells, resulting in reduced growth rates compared to an untreated control culture (Figure 1). Fluorescence staining of proteins additionally allowed for quantification of protein amounts actually present in the samples. Proteins with a pI of 4 to 7 were separated on 2D gels, resulting in the detection of 1167 spots with assigned protein identification. This led to the quantification of 425 proteins in all conditions examined, of which 107 proteins were significantly altered in either amount or synthesis after treatment with at least one of the antibiotics tested (Supplementary Tables S1 and S2).

Essential proteins represent possible targets of antimicrobial drugs. Until now, a list of putative essential proteins in C. difficile has only been published for strain R20291, which comprises 377 proteins [32]. Hence, homologous genes in C. difficile 630 $\Delta \mathrm{erm}$ were identified using Proteinortho [33] and their protein amount and synthesis rate were checked in the dataset provided in this study. Indeed, 103 homologous proteins could be quantified of which 20 were significantly altered in either synthesis or protein amount. Among all differentially regulated proteins ribosomal proteins, tRNA ligases and enzymes involved in cell wall synthesis could be identified (Table 1). Those regulated essential proteins may represent (direct or indirect) targets of the antibiotics examined which are affected independently and regardless of the primary mode of action of those antimicrobial compounds.

\subsection{Proteomic Signatures for Vancomycin, Metronidazole, and Fidaxomicin Stress}

Treatment of bacterial cells with antibiotics causes physiological responses aimed at elimination of cellular damage and prevention of further impairment by the drug. Thereby the pattern of cellular responses is highly dependent on the antibiotic target and mechanism of action [34]. Hence, every antibiotic treatment will result in a proteomic signature indicating the mode of action of this drug. In this study, separation of proteins by 2D PAGE did not only allow to quantify changes in protein synthesis and accumulated protein amounts but also supports the deduction and visualization of proteomic signatures during treatment of $C$. difficile with vancomycin, metronidazole, or fidaxomicin (Figure 2). The results indicate very specific cellular responses to the antibiotics, as more than $87 \%$ of the differentially expressed proteins exhibit changes only after treatment with one of the antimicrobial compounds. This results in proteomic signatures, which are very specific for each drug. In detail, eight signature proteins could be deduced for vancomycin stress, and 47 and 39 signature proteins were detected for metronidazole and fidaxomicin treatment, respectively (Table 2). However, a small number of proteins were regulated by more than one antimicrobial compound. Specifically, the responses to metronidazole and fidaxomicin showed more similarities when compared to each other than when compared to vancomycin stress (Figure 2). Indeed, along with the reduced growth rate caused by treatment with both antimicrobials, fidaxomicin and metronidazole, altered amounts of proteins involved in DNA metabolism, transcription or cell wall metabolism were detected. 
Table 1. This table lists all proteins which were significantly altered ( $p$-value $<0.05$, average $\log _{2}$ fold change $>|0.8|$ ) in either synthesis or amount after treatment of $C$. difficile $630 \Delta \mathrm{erm}$ with sublethal concentrations of vancomycin (VAN), metronidazole (MTZ) or fidaxomicin (FDX), respectively. A protein was assumed to be essential when it showed reciprocal best hits to its homologue in C. difficile R20291 after an analysis with Proteinortho [33].

\begin{tabular}{|c|c|c|c|c|c|c|c|c|c|}
\hline \multirow{3}{*}{ Accession Number } & \multirow{3}{*}{ Protein Name } & \multirow{3}{*}{ R20291-Homologue } & \multirow{3}{*}{ Function } & \multicolumn{6}{|c|}{ Significantly Changed $\left(p<0.05, \log _{2}\right.$ Fold Change $\left.>|0.8|\right)$} \\
\hline & & & & \multicolumn{3}{|c|}{ Amount } & \multicolumn{3}{|c|}{ Synthesis } \\
\hline & & & & VAN & MTZ & FDX & VAN & MTZ & FDX \\
\hline CDIF630erm_01408 & $\operatorname{RimM}$ & CDR20291_1095 & 16S rRNA processing protein RimM & $\mathrm{x}$ & $\mathrm{x}$ & & & $\mathrm{x}$ & \\
\hline CDIF630erm_03522 & DapB1 & CDR20291_3086 & 4-hydroxy-tetrahydrodipicolinate reductase & & $\mathrm{x}$ & $\mathrm{x}$ & & $\mathrm{x}$ & $\mathrm{x}$ \\
\hline CDIF630erm_03707 & MurB & CDR20291_3224 & UDP- $N$-acetylenolpyruvoyl-glucosamine reductase & & $\mathrm{x}$ & $\mathrm{x}$ & & & \\
\hline CDIF630erm_02841 & RpoZ & CDR20291_2474 & DNA-directed RNA polymerase subunit omega & & $\mathrm{x}$ & $\mathrm{x}$ & & & \\
\hline CDIF630erm_01509 & CDIF630erm_01509 & CDR20291_1195 & putative pyridoxine kinase & & $\mathrm{x}$ & & & & \\
\hline CDIF630erm_02877 & CDIF630erm_02877 & CDR20291_2507 & putative pyridoxal $5^{\prime}$-phosphate-dependent enzyme & & $\mathrm{x}$ & & & & \\
\hline CDIF630erm_02708 & GrpE & CDR20291_2355 & stress protein (HSP-70 cofactor) & & $\mathrm{x}$ & & & & \\
\hline CDIF630erm_04005 & MnmG & CDR20291_3535 & tRNA uridine 5-carboxymethyl-aminomethyl modification enzyme & & $\mathrm{x}$ & & & & \\
\hline CDIF630erm_03828 & Prs & CDR20291_3351 & ribose-phosphate pyrophosphokinase & & $\mathrm{x}$ & & & & \\
\hline CDIF630erm_00260 & SecA1 & CDR20291_0142 & protein translocase subunit SecA1 & & $\mathrm{x}$ & & & & \\
\hline CDIF630erm_01292 & MreB2 & CDR20291_0982 & rod shape-determining protein MreB & & & $\mathrm{x}$ & & & $\mathrm{x}$ \\
\hline CDIF630erm_03832 & MurC & CDR20291_3355 & UDP- $N$-acetylmuramate-L-alanine ligase & & & $\mathrm{x}$ & & $\mathrm{x}$ & \\
\hline CDIF630erm_03585 & Pgi & CDR20291_3146 & glucose-6-phosphate isomerase & & & & $\mathrm{x}$ & & \\
\hline CDIF630erm_02574 & GuaB & CDR20291_2224 & IMP dehydrogenase & & & & & $\mathrm{x}$ & \\
\hline CDIF630erm_03992 & RpsF & CDR20291_3523 & $30 \mathrm{~S}$ ribosomal protein $\mathrm{S} 6$ & & & & & $\mathrm{x}$ & \\
\hline CDIF630erm_00160 & Adk & CDR20291_0088 & adenylate kinase & & & & & & $\mathrm{x}$ \\
\hline CDIF630erm_02772 & LeuS & CDR20291_2410 & leucine-tRNA ligas & & & & & & $\mathrm{x}$ \\
\hline CDIF630erm_03872 & LysS & CDR20291_3389 & lysine-tRNA ligase & & & & & & $\mathrm{x}$ \\
\hline CDIF630erm_00461 & CDIF630erm_00461 & CDR20291_0338 & cobalt-dependent inorganic pyrophosphatase & & & & & & $\mathrm{x}$ \\
\hline
\end{tabular}



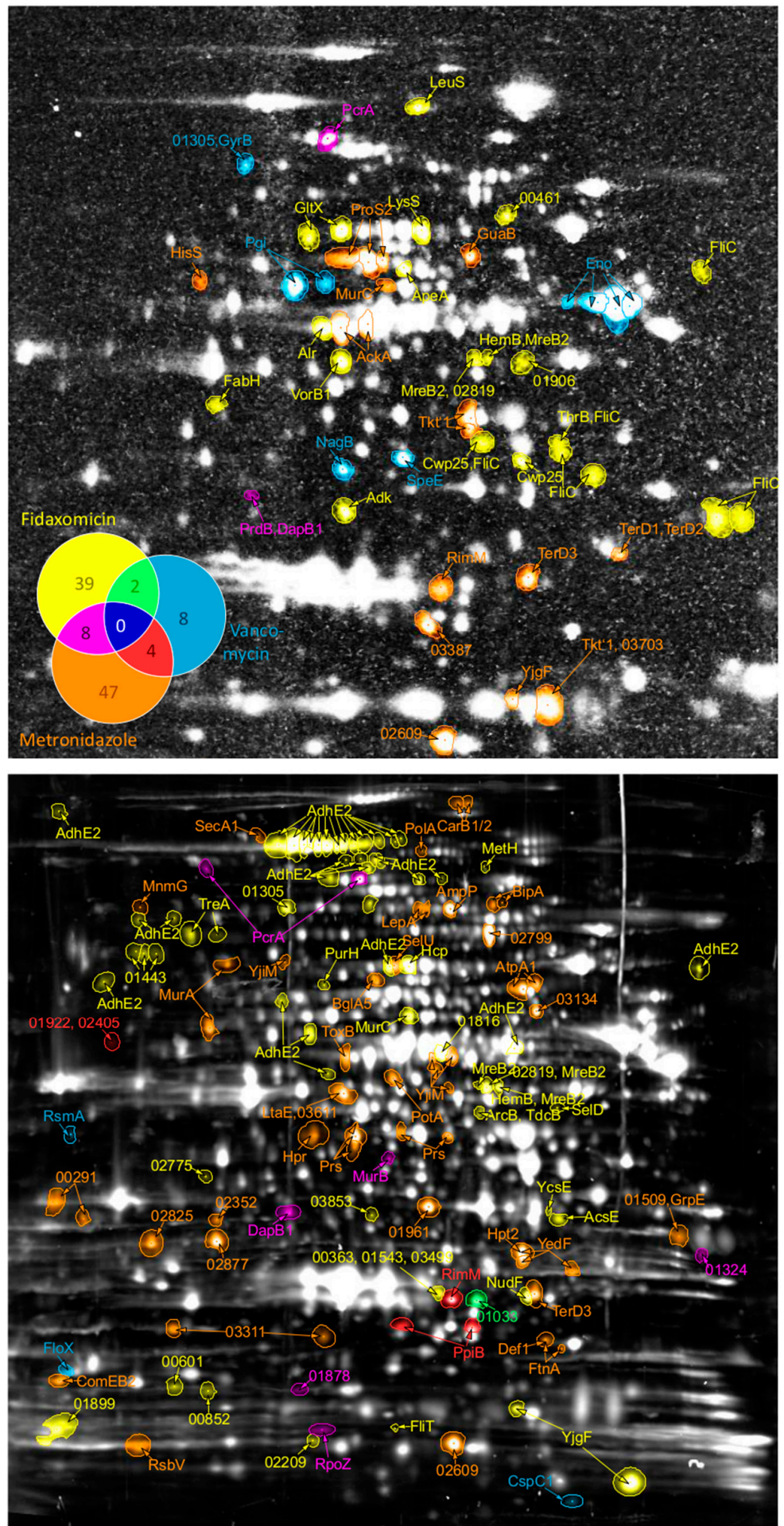

Figure 2. Protein pattern of antibiotic signatures in C. difficile. Spots of significantly changed proteins are indicated on the autoradiogram (upper part) for proteins with altered protein synthesis rate or on the 2D gel stained for total protein amount (lower part) for proteins with significantly altered amounts. Protein names (if available) or accession numbers (excluding "CDIF630erm_") according to Dannheim et al. [31] were used as labels. The colors as assigned in the Venn-diagram (top-left) indicate after which antibiotic treatment the proteomic response became detectable. Numbers in the Venn-diagram represent the quantity of proteins altered in synthesis rate or abundance after antibiotic treatment. 
Table 2. This table lists all proteins uniquely regulated after treatment with the antibiotic they were assigned to (vancomycin (VAN), metronidazole (MTZ), fidaxomicin (FDX)). Column "regulation" indicates up $(\uparrow)$ or downregulation $(\downarrow)$ in both sampling points (10 and $30 \mathrm{~min})$ or changing regulation during adaptation $(\uparrow \downarrow)$.

\begin{tabular}{|c|c|c|c|c|}
\hline Antibiotic & Accession Number & Protein Name & Function & Regulation \\
\hline VAN & CDIF630erm_00928 & FloX & putative flavodoxin & $\uparrow$ \\
\hline VAN & CDIF630erm_00005 & GyrB & DNA gyrase subunit B & $\uparrow$ \\
\hline VAN & CDIF630erm_01147 & NagB & glucosamine-6-phosphate deaminase & $\uparrow$ \\
\hline VAN & CDIF630erm_03585 & Pgi & glucose-6-phosphate isomeras & $\uparrow$ \\
\hline VAN & CDIF630erm_02205 & CspC1 & major cold shock protein CspC & $\downarrow$ \\
\hline VAN & CDIF630erm_03462 & Eno & enolase & $\downarrow$ \\
\hline VAN & CDIF630erm_01010 & SpeE & spermidine synthase & $\downarrow$ \\
\hline VAN & CDIF630erm_03837 & RsmA & ribosomal RNA small subunit methyltransferase A & $\uparrow \downarrow$ \\
\hline MTZ & CDIF630erm_03239 & AtpA1 & V-type ATP synthase subunit A & $\uparrow$ \\
\hline MTZ & CDIF630erm_03421 & BglA5 & 6-phospho-beta-glucosidase & $\uparrow$ \\
\hline MTZ & CDIF630erm_03910 & CarB1 & carbamoyl-phosphate synthase large subunit & $\uparrow$ \\
\hline MTZ & CDIF630erm_03912 & CarB2 & carbamoyl-phosphate synthase large subunit & $\uparrow$ \\
\hline MTZ & CDIF630erm_00291 & CDIF630erm_00291 & ABC-type transport system, ATP-binding protein & $\uparrow$ \\
\hline MTZ & CDIF630erm_02352 & CDIF630erm_02352 & $\begin{array}{l}\text { putative multiprotein-complex assembly TPR } \\
\text { repeat-containing protein }\end{array}$ & $\uparrow$ \\
\hline MTZ & CDIF630erm_03387 & CDIF630erm_03387 & PTS system, glucose-like IIA component & $\uparrow$ \\
\hline MTZ & CDIF630erm_03611 & CDIF630erm_03611 & $\begin{array}{c}\text { putative D-isomer specific } \\
\text { 2-hydroxyacid dehydrogenase }\end{array}$ & $\uparrow$ \\
\hline MTZ & CDIF630erm_03703 & CDIF630erm_03703 & putative hydrolase, NUDIX family & $\uparrow$ \\
\hline MTZ & CDIF630erm_03788 & ComEB2 & deoxycytidylate deaminase & $\uparrow$ \\
\hline MTZ & CDIF630erm_01952 & Def1 & peptide deformylase & $\uparrow$ \\
\hline MTZ & CDIF630erm_02427 & Ftn $\mathrm{A}$ & bacterial non-heme ferritin & $\uparrow$ \\
\hline MTZ & CDIF630erm_03003 & HisS & histidine-tRNA ligase & $\uparrow$ \\
\hline MTZ & CDIF630erm_01131 & $\mathrm{Hpr}$ & hydroxypyruvate reductase & $\uparrow$ \\
\hline MTZ & CDIF630erm_02713 & LepA & elongation factor 4 & $\uparrow$ \\
\hline MTZ & CDIF630erm_02845 & LtaE & low specificity L-threonine aldolase & $\uparrow$ \\
\hline MTZ & CDIF630erm_04005 & MnmG & $\begin{array}{l}\text { tRNA uridine 5-carboxymethylaminomethyl } \\
\text { modification enzyme }\end{array}$ & $\uparrow$ \\
\hline MTZ & CDIF630erm_01275 & PolA & DNA polymerase I (POLI) & $\uparrow$ \\
\hline MTZ & CDIF630erm_01160 & PotA & $\begin{array}{c}\text { ABC-type transport system, spermidine/putrescine } \\
\text { ATP-binding protein }\end{array}$ & $\uparrow$ \\
\hline MTZ & CDIF630erm_00260 & SecA1 & protein translocase subunit SecA1 & $\uparrow$ \\
\hline MTZ & CDIF630erm_01481 & SelU & tRNA 2-selenouridine synthase & $\uparrow$ \\
\hline MTZ & CDIF630erm_01811 & TerD1 & tellurium resistance protein $\mathrm{Ter} D$ & $\uparrow$ \\
\hline MTZ & CDIF630erm_02559 & Tkt'1 & transketolase, C-terminal section & $\uparrow$ \\
\hline MTZ & CDIF630erm_00773 & ToxB & toxin $B$ & $\uparrow$ \\
\hline MTZ & CDIF630erm_01943 & YjiM & putative 2-hydroxyacyl-CoA dehydratase & $\uparrow$ \\
\hline MTZ & CDIF630erm_01323 & AckA & acetate kinase & $\downarrow$ \\
\hline MTZ & CDIF630erm_02495 & AmpP & xaa-pro aminopeptidase & $\downarrow$ \\
\hline MTZ & CDIF630erm_02343 & BipA & GTP-binding protein BipA & $\downarrow$ \\
\hline MTZ & CDIF630erm_01509 & CDIF630erm_01509 & putative pyridoxine kinase & $\downarrow$ \\
\hline MTZ & CDIF630erm_01961 & CDIF630erm_01961 & putative phenylalanyl-tRNA synthetase beta chain & $\downarrow$ \\
\hline MTZ & CDIF630erm_02609 & CDIF630erm_02609 & DsrEFH-like protein & $\downarrow$ \\
\hline MTZ & CDIF630erm_02799 & CDIF630erm_02799 & hypothetical protein & $\downarrow$ \\
\hline MTZ & CDIF630erm_02825 & CDIF630erm_02825 & putative nitroreductase-like oxidoreductase & $\downarrow$ \\
\hline MTZ & CDIF630erm_02877 & CDIF630erm_02877 & putative pyridoxal 5'-phosphate-dependent enzyme & $\downarrow$ \\
\hline MTZ & CDIF630erm_02881 & CDIF630erm_02881 & ACT domain-containing protein & $\downarrow$ \\
\hline MTZ & CDIF630erm_03134 & CDIF630erm_03134 & putative modulator of DNA gyrase, peptidase U62 & $\downarrow$ \\
\hline MTZ & CDIF630erm_03311 & CDIF630erm_03311 & PTS system, arabinose-specific IIA component & $\downarrow$ \\
\hline MTZ & CDIF630erm_02708 & GrpE & protein (HSP-70 cofactor) & $\downarrow$ \\
\hline
\end{tabular}


Table 2. Cont

\begin{tabular}{|c|c|c|c|c|}
\hline Antibiotic & Accession Number & Protein Name & Function & Regulation \\
\hline MTZ & CDIF630erm_02574 & GuaB & IMP dehydrogenase & $\downarrow$ \\
\hline MTZ & CDIF630erm_03527 & Hpt2 & hypoxanthine phosphoribosyltransferase & $\downarrow$ \\
\hline MTZ & CDIF630erm_00239 & MurA & $\begin{array}{l}\text { UDP-N-acetylglucosamine } \\
\text { 1-carboxyvinyltransferase }\end{array}$ & $\downarrow$ \\
\hline MTZ & CDIF630erm_00113 & ProS2 & proline-tRNA ligase & $\downarrow$ \\
\hline MTZ & CDIF630erm_03828 & Prs & ribose-phosphate pyrophosphokinase & $\downarrow$ \\
\hline MTZ & CDIF630erm_03992 & RpsF & $30 \mathrm{~S}$ ribosomal protein $\mathrm{S} 6$ & $\downarrow$ \\
\hline MTZ & CDIF630erm_00009 & RsbV & anti-sigma factor antagonist & $\downarrow$ \\
\hline MTZ & CDIF630erm_03996 & YedF & selenium metabolism protein YedF & $\downarrow$ \\
\hline MTZ & CDIF630erm_01998 & TerD4 & tellurium resistance protein TerD & $\uparrow \downarrow$ \\
\hline FDX & CDIF630erm_00843 & AcsE & $\begin{array}{l}\text { 5-methyltetrahydrofolate I corrinoid/iron-sulfur } \\
\text { protein co-methyltransferase }\end{array}$ & $\uparrow$ \\
\hline FDX & CDIF630erm_03250 & AdhE2 & aldehyde-alcohol dehydrogenase & $\uparrow$ \\
\hline FDX & CDIF630erm_00160 & Adk & adenylate kinase & $\uparrow$ \\
\hline FDX & CDIF630erm_00657 & ArcB & Delta (1)-pyrroline-2-carboxylate reductase & $\uparrow$ \\
\hline FDX & CDIF630erm_00363 & CDIF630erm_00363 & phosphothreonine phosphatase & $\uparrow$ \\
\hline FDX & CDIF630erm_00461 & CDIF630erm_00461 & cobalt-dependent inorganic pyrophosphatase & $\uparrow$ \\
\hline FDX & CDIF630erm_01443 & CDIF630erm_01443 & ribonuclease J family protein & $\uparrow$ \\
\hline FDX & CDIF630erm_01543 & CDIF630erm_01543 & transcriptional regulator, GntR family & $\uparrow$ \\
\hline FDX & CDIF630erm_01816 & CDIF630erm_01816 & putative tellurite associated resistance protein & $\uparrow$ \\
\hline FDX & CDIF630erm_01906 & CDIF630erm_01906 & uncharacterized protein & $\uparrow$ \\
\hline FDX & CDIF630erm_02819 & CDIF630erm_02819 & $\begin{array}{l}\text { PTS system, mannosylglycerate-specific } \\
\text { IIA component }\end{array}$ & $\uparrow$ \\
\hline FDX & CDIF630erm_03499 & CDIF630erm_03499 & putative nitroreductase-like oxidoreductase & $\uparrow$ \\
\hline FDX & CDIF630erm_03853 & CDIF630erm_03853 & putative deoxyribonuclease & $\uparrow$ \\
\hline FDX & CDIF630erm_00963 & Cwp25 & putative cell wall-binding protein & $\uparrow$ \\
\hline FDX & CDIF630erm_01328 & $\mathrm{FabH}$ & beta-ketoacyl-[acyl-carrier-protein] synthase 3 & $\uparrow$ \\
\hline FDX & CDIF630erm_00361 & FliC & flagellin C & $\uparrow$ \\
\hline FDX & CDIF630erm_00114 & GltX & glutamate-tRNA ligase & $\uparrow$ \\
\hline FDX & CDIF630erm_02400 & Hсp & hydroxylamine reductase & $\uparrow$ \\
\hline FDX & CDIF630erm_03727 & HemB & delta-aminolevulinic acid dehydratase & $\uparrow$ \\
\hline FDX & CDIF630erm_02772 & LeuS & leucine-tRNA ligase & $\uparrow$ \\
\hline FDX & CDIF630erm_03872 & LysS & lysine-tRNA ligase & $\uparrow$ \\
\hline FDX & CDIF630erm_03918 & MetH & methionine synthase & $\uparrow$ \\
\hline FDX & CDIF630erm_01292 & MreB2 & rod shape-determining protein $\mathrm{MreB}$ & $\uparrow$ \\
\hline FDX & CDIF630erm_01369 & NudF & ADP-ribose pyrophosphatase & $\uparrow$ \\
\hline FDX & CDIF630erm_00345 & PurH & $\begin{array}{c}\text { bifunctional } \\
\text { phosphoribosylaminoimidazolecarboxamide } \\
\text { formyltransferase/IMP cyclohydrolase }\end{array}$ & $\uparrow$ \\
\hline FDX & CDIF630erm_02743 & SelD & selenide, water dikinase & $\uparrow$ \\
\hline FDX & CDIF630erm_02763 & $\mathrm{TdcB}$ & threonine dehydratase & $\uparrow$ \\
\hline FDX & CDIF630erm_02348 & ThrB & homoserine kinase & $\uparrow$ \\
\hline FDX & CDIF630erm_03374 & TreA & trehalose-6-phosphate hydrolase & $\uparrow$ \\
\hline FDX & CDIF630erm_00231 & VorB1 & $\begin{array}{l}\text { 3-methyl-2-oxobutanoate dehydrogenase } \\
\text { (ferredoxin), beta subunit }\end{array}$ & $\uparrow$ \\
\hline FDX & CDIF630erm_02749 & YcsE & $\begin{array}{l}\text { putative 5-amino-6-(5-phospho-D-ribitylamino) } \\
\text { uracil phosphatase }\end{array}$ & $\uparrow$ \\
\hline FDX & CDIF630erm_03774 & Alr & alanine racemase & $\downarrow$ \\
\hline FDX & CDIF630erm_01216 & ApeA & aminopeptidase ApeA & $\downarrow$ \\
\hline FDX & CDIF630erm_00601 & CDIF630erm_00601 & transcriptional regulator, MarR family & $\downarrow$ \\
\hline FDX & CDIF630erm_00852 & CDIF630erm_00852 & uncharacterized protein & $\downarrow$ \\
\hline FDX & CDIF630erm_01899 & CDIF630erm_01899 & uncharacterized protein, UPF0145 family & $\downarrow$ \\
\hline FDX & CDIF630erm_02209 & CDIF630erm_02209 & uncharacterized protein, AhpD-like & $\downarrow$ \\
\hline FDX & CDIF630erm_02775 & CDIF630erm_02775 & putative hydrolase & $\downarrow$ \\
\hline FDX & CDIF630erm_00360 & FliT & flagellar protein FliT & $\downarrow$ \\
\hline
\end{tabular}




\subsection{Proteomic Adaptation to Vancomycin}

The proposed mechanism of action for vancomycin includes the binding of the antimicrobial molecule to an essential precursor in cell wall synthesis, which results in a slow killing rate of this antibiotic and causes a bacteriostatic effect in C. difficile [35]. In the dataset presented here, only 14 proteins were significantly altered in either synthesis or amount after treatment (Supplementary Tables S1 and S2), of which eight proteins represent signature proteins for vancomycin (Table 2). Changes in synthesis rate or abundance could be detected for proteins involved in intermediary metabolism as well as in the regulation of other proteins' synthesis or fate (according to TIGRFAMs [36], Figure 3). However, the number of differentially expressed proteins during vancomycin stress is strikingly lower compared to the number of proteins altered after treatment with metronidazole or fidaxomicin, respectively.

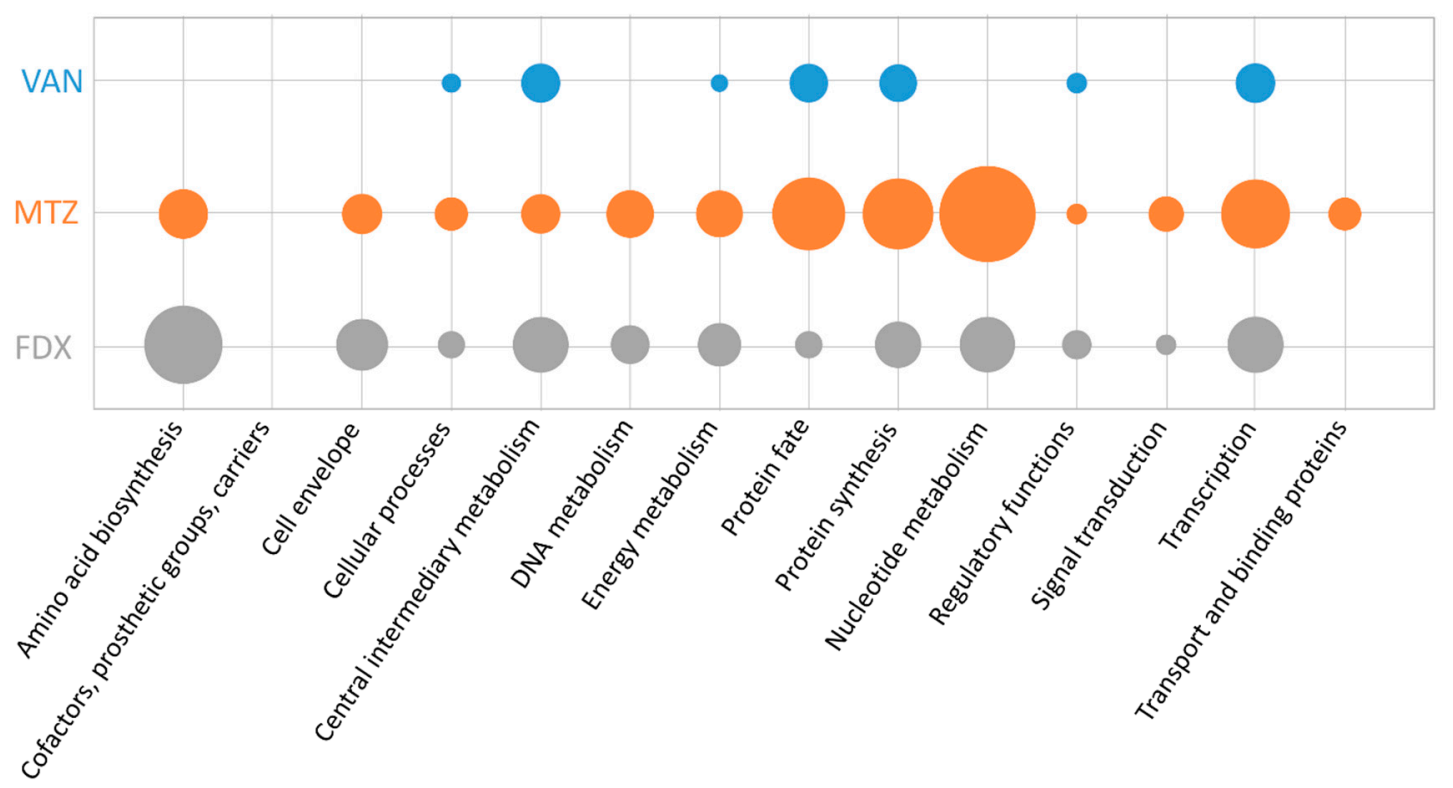

Figure 3. Functional categorization of significantly altered proteins according to TIGRFAMs [36]. The number of significantly altered proteins after treatment with vancomycin (VAN, blue), metronidazole (MTZ, orange), or fidaxomicin (FDX, grey) correlated to the total number of proteins in the respective functional category are represented by the circle's size. Hence, enriched categories are represented by bigger circles.

Signature proteins for vancomycin stress fulfil various cellular functions, suggesting that the differential expression of those proteins in the early adaptation phase is rather a secondary than a direct effect of vancomycin treatment. However, this study still revealed signature proteins directly related to the mode of action of vancomycin, namely the glucosamine-6-phosphate deaminase (CDIF630erm_01147), the glucose-6-phosphate isomerase Pgi (CDIF630erm_03585), both providing precursors for cell wall biosynthesis and the peptidyl-prolyl cis-trans isomerase B PpiB (CDIF630erm_00459) involved in protein homeostasis of the cell envelope.

\subsection{Proteomic Adaptation to Metronidazole}

The antimicrobial function of metronidazole is mediated by the disruption of DNA in anaerobic cells that results in inhibition of nucleic acid synthesis. In the study presented here, 59 proteins of $C$. difficile were significantly altered in either synthesis or amount after metronidazole stress (Supplementary Tables S1 and S2). A significant fraction of those proteins fulfil functions in intermediary, energy, or nucleotide metabolism or are assigned to the functional categories "protein synthesis" and "protein fate". Moreover, several proteins with changed amount or regulated synthesis 
after metronidazole stress play a role in amino acid metabolism, transcription or cellular processes including adaptation to atypical conditions (Figure 3).

Due to the proposed mode of action of metronidazole, it could be expected to find differentially regulated proteins involved in DNA replication, recombination, and repair. Indeed, the deoxycytidylate deaminase ComEB2 (CDIF630erm_03788), DNA helicase PcrA (CDIF630erm_00456), DNA polymerase I PolA (CDIF630erm_01275), and carbamoyl-phosphate synthase CarB (CarB1 (CDIF630erm_03910), CarB2 (CDIF630erm_03912), identified in the same spot on the 2D gel) were significantly more abundant after treatment with metronidazole.

Additionally, the proteomic signature of metronidazole stress is characterized by alterations of proteins involved in protein biosynthesis and protein fate pointing to a substantial reprogramming of protein expression during adaptation to metronidazole. In this context it should be noted that 13 tRNA ligases (AlaS, AsnC, GlnS, GltX, GlyQ, GlyS, HisS, IleS LeuS, LysS, MetG, ThrS, ValS) were found to be increased more than 1.75-fold in either protein amount or protein synthesis during adaptation. The energy necessary for the active reorganization of the proteome is provided by the activation of the energy metabolism in the early adaptation phase as supported by the detection of differentially expressed proteins assigned to this functional category.

The specificity of metronidazole against anaerobic microbes is caused by nitroreductases and oxidoreductases that are present in these bacteria. Both enzyme classes activate metronidazole via reduction resulting in unstable and/or less reduced intermediates that damage DNA by strand breakage, helix destabilization, and helix unwinding leading to cell death [37]. Although 43 nitroreductases or oxidoreductases have been annotated in the $C$. difficile genome, only ten of those enzymes could be quantified in this study and none of them were significantly altered in either protein synthesis or amount.

Furthermore, it is worth mentioning that a significantly increased protein synthesis was detected for the tellurium resistance protein TerD, putatively involved in detoxification of the antimicrobial. The tellurium resistance protein is represented by its paralogues TerD1 (CDIF630erm_01811) and TerD4 (CDIF630erm_01998) $30 \mathrm{~min}$ after addition of metronidazole to the C.difficile culture. However, although no increase in protein amount was detectable during the early phase of adaptation, changes in the protein amount of TerD in later time points after metronidazole stress can be expected based on protein synthesis data.

\subsection{Proteomic Adaptation to Fidaxomicin}

Fidaxomicin functions by binding to the DNA strand-RNA polymerase-complex, which prevents successful mRNA synthesis. In the current study 49 proteins where significantly altered in either amount or synthesis rate after treatment with fidaxomicin (Supplementary Tables S1 and S2). Thereby differentially regulated proteins can be assigned to a variety of biological functions, of which amino acid and protein biosynthesis, transcription, nucleotide metabolism, cell motility and functions in the cell envelope are most prominent (Figure 3). More specifically, ArcB (CDIF630erm_00657), DapB1 (CDIF630erm_03522), MetH (CDIF630erm_03918), TdcB (CDIF630erm_02763), and ThrB (CDIF630erm_02348), involved in synthesis of aspartate, glutamate, methionine, threonine, or serine, respectively, have been found to be upregulated in response to fidaxomicin.

Due to the mode of action of fidaxomicin, it can be expected that proteins with functions in transcription and translation are regulated when cells are facing this drug. This hypothesis can be supported by the data presented here. While YigF (CDIF630erm_02762), involved in RNA degradation, is downregulated, other proteins with functions during transcription and translation, like RpoZ (CDIF630erm_02841), or various tRNA ligases (LeuS (CDIF630erm_02772), LysS (CDIF630erm_03872), GltX (CDIF630erm_00114)) were found to be significantly upregulated.

Moreover, the current study suggests that treatment of $C$. difficile with fidaxomicin alters processes in the cell envelope. In more detail, Alr (CDIF630erm_03774), Cwp25 (CDIF630erm_00963), 
MreB2 (CDIF630erm_01292), MurB and MurC (CDIF630erm_03707 and CDIF630erm_03832) were differentially expressed during fidaxomicin stress.

Additionally, it should be mentioned that treatment of $C$. difficile with fidaxomicin alters the amount of two transcriptional regulators that have not been further characterized. The regulatory protein of the GntR-family (CDIF630erm_01543) was found with increased amounts as soon as 10 minutes after application of fidaxomicin to the bacterial culture. In contrast, the transcriptional regulator of the MarR-family (CDIF630erm_00601) was detected with lowered amounts during treatment.

\section{Discussion}

\subsection{Technical Aspects of Gel-Based Approaches to Derive Antibiotic Signature}

Due to the increasing incidence of antibiotic-resistant $C$. difficile it is of great importance to understand the detailed adaptation mechanisms of this widespread pathogen to antibiotic treatment. In this context the analysis of proteins is of great interest as these molecules catalyze crucial metabolic reactions as well as cellular processes necessary during pathogenesis. Indeed, it is the proteome which translates the genome sequence of an organism into cellular functions and structures, and therefore proteins can be regarded as the main players of life. Therefore, proteomics allows to monitor cellular adaptation to changing environmental conditions, which may also give hints to the mechanism of action of different antibiotics. In order to investigate the proteomic adaptation of $C$. difficile to commonly used antibiotics in a comprehensive and sensitive manner, it is beneficial to detect not only changes in protein amount, but also in protein synthesis. The availability of quantitative data on protein amount and protein synthesis in this study enabled the sensitive detection of alterations in TerD synthesis after metronidazole stress. Indeed, the induced synthesis of the putatively detoxifying enzymes TerD1 and TerD4 could be detected already during early time points of adaptation, whereas it remains elusive if detectable changes in protein amounts can be observed later after antibiotic stress. An example of limitation for a study combining investigations on both protein synthesis rate and protein amount is AdhE2 (CDIF630erm_03250), of which increased amounts could be detected after fidaxomicin stress. However, the fact that no radioactive signal could be detected for this spot hints to an insufficient labeling of the newly synthesized AdhE2.

The preferred and still most widely used technique for visualization and quantification of protein synthesis is pulse-labeling with radioactive amino acids [23-25]. However, a prerequisite for successful labeling of newly synthesized proteins is the sufficient incorporation of the radioactive label in the proteins. Labeling with ${ }^{35} \mathrm{~S}$ works best, if neither methionine nor any other unlabeled sulfur source is present in the medium during growth. Moreover, labeling with radioactive methionine is most efficient if all methionine offered is incorporated into the proteins synthesized during the investigated time span. However, both methionine and cysteine are required for $C$. difficile growth in any medium and both amino acids are not only used for protein biosynthesis but also for energy metabolism [38]. Therefore, radioactive labeling efficiencies had to be evaluated and the protocols for pulse-labeling of $C$. difficile had to be adapted. The modified method applied in this study opens the possibility to investigate changes in protein synthesis in $C$. difficile with radioactive pulse-labeling. One disadvantage, however, is the need for doses of radioactivity to label sufficient protein amounts for a single 2D gel ten times higher than for comparable experiments in Bacillus subtilis [39]. In order to correct for proteomic effects that are caused by damage to the DNA, which might be introduced by the high amount of radioactivity, the control cultures have also been labeled with ${ }^{35} \mathrm{~S}$-methionine. Hence, all differences in either protein amount or protein synthesis rate described in this study are solely caused by the antibiotic treatment.

In order to be able to compare protein adaptation after treatment with several antimicrobial agents it is necessary to select appropriate sampling points. However, if the mode of action of the antibiotics examined differs significantly it is possible that the time span necessary to detect a proteomic or even a phenotypic adaptation varies. In this study, the number of differentially regulated proteins during 
vancomycin stress is strikingly lower compared to the number of proteins altered after treatment with metronidazole or fidaxomicin. Although the sampling points selected for the experiments seem to be suitable for metronidazole and fidaxomicin stress, they might be too early after vancomycin stress to detect proteomic changes. This idea is supported by the fact that for none of the proteins exhibiting differential protein synthesis rates after treatment with vancomycin changes in protein amount could be detected. Moreover, in a study on vancomycin stress in Staphylococcus aureus the authors only took samples $100 \mathrm{~min}$ after adding vancomycin to the bacterial culture in order to detect adaptive changes in protein amount [40]. In this study, growth inhibitory effects became visible only after $30 \mathrm{~min}$ of treatment. Indeed, the delayed response to the antibiotic was also observed in C. difficile, where reduction in growth rate started $60 \mathrm{~min}$ after addition of vancomycin. However, the current study aimed not only at monitoring changes in protein amount but also at examining the proteomic adaptation on the level of protein synthesis, which is usually an immediate cellular response to perturbations. In the case of metronidazole and fidaxomicin stress, sampling after $60 \mathrm{~min}$ of treatment might be too late to monitor the early proteomic responses to those antibiotics. Hence, the selection of early sampling points was reasonable as changes in protein synthesis within the first $30 \mathrm{~min}$ of stress were expected for all three antibiotics.

Additionally, the study on S. aureus during vancomycin treatment revealed the most striking impact on the amount of proteins localized in or attached to the cell wall [40]. However, those proteins cannot be covered in a 2D gel-based study, as the quantification of this protein class requires specific protein enrichment and sample preparation workflows that are incompatible with 2D PAGE. The same holds true for a more detailed analysis of adaptational processes in the cell envelope after treatment with fidaxomicin. Hence, it remains speculative if the putative reorganization of cell surface components results from attempts to repel the antimicrobial compound or if the data hint to independent or even secondary effects of fidaxomicin treatment. Further research may provide more details on the impact of fidaxomicin and vancomycin to the cell envelope of $C$. difficile. Moreover, proteins associated to the bacterial cell surface or secreted into the environment represent important virulence factors and may also be directly involved in the recognition, targeting, and detoxification of antimicrobials. Hence, it may also be useful to complement the available data sets in follow-up studies with comprehensive views on other subcellular proteome fractions than the soluble proteins in the cell.

\subsection{Protein Signatures in Antibiotic Research in C. difficile}

A vast collection of comprehensive knowledge on bacterial adaptation to antibiotic stress is a prerequisite to understand the complex interaction of bacteria, drugs, and environment. This is even more important in pathogenic bacteria where this information is necessary to discover activities that reduce the development of resistance or prevent resistant strains from spreading. However, most data available focus on well described model organisms like Escherichia coli, B. subtilis, or S. aureus.

The largest publicly available compendium to date contains proteome profiles of the Gram-positive model organism B. subtilis. These data are, like in this study, based on 2D PAGE, and contain proteome patterns of B. subtilis treated with 30 different antimicrobial compounds [25]. Such comprehensive datasets enabled the authors to group the proteomic adaptation according to the antibiotics examined. Interestingly, the grouping of antibiotics based on the previously described responses of E. coli [41] was the same as the grouping based on the responses of B. subtilis, while the marker (responder) proteins were species-specific [34]. This may also hold true in other bacteria such as $C$. difficile. Hence, the compilation of $C$. difficile proteomic signatures in this and following studies will facilitate a successful grouping of different physiological strategies to counteract antibiotics, which will subsequently allow for the deduction of the mechanism of action of new antibiotics aiming at treating CDI.

In addition to the opportunity to group the proteomics adaptation to antibiotic treatments according to the (putative) mode of action, the deduction of signature proteins enhances the knowledge on resistance development and spreading as it defines specific responses to antimicrobial compounds. 
Indeed, the expression of only a little number of proteins is sufficient to render a microorganism resistant to a particular antibiotic. Such proteins function as efflux pumps, antibiotic-degrading or modifying enzymes, or represent a mutated antibiotic target. Most often, the antibiotic-regulated proteins can be linked directly or indirectly to the physiological state of the bacterial cell during antibiotic treatment. Hence, it was surprising that only very few signature proteins identified in this study seem to be directly linked to the drug applied. However, proteins regulated after antibiotic treatment may not only be directly involved in compensating for the loss of a particular function or in repairing the damage inflicted by an antibiotic but could also be indirectly connected to the anticipated mechanism of action of a specific antimicrobial. Moreover, the unique regulation of signature proteins in response to a particular antibiotic may point to unexpected targets that may also be prone to variations during resistance development.

\subsection{Response to Metronidazole in Other Anaerobic Bacteria}

Previous investigations on the adaptation of C. difficile NAP1, Bacterioides fragilis, and Helicobacter pylori to metronidazole revealed the involvement of various protein groups in the detoxification of metronidazole and the development of resistance [42-45]. Those proteins are involved in DNA replication, recombination and repair, protein synthesis and regulation of protein stability, or represent stress proteins, or nitroreductases and oxidoreductases.

In a microarray analysis of the transcriptional responses of $C$. difficile grown in the presence of a sub-inhibitory concentration of metronidazole, it has been reported that the genes coding for ribosomal proteins, subunits of the RNA polymerase, elongation and translation factors as well as the majority of tRNA ligases were coordinately upregulated [43]. Although the upregulation of tRNA ligases can also be observed in C. difficile, the influence of metronidazole treatment on other proteins involved in protein synthesis was not that clear. In more detail, five proteins of the large ribosomal subunit and six proteins of the small ribosomal subunit, as well as three subunits of the RNA polymerase (RpoA, RpoB, RpoZ) have been quantified in this proteomics study. However, none of those proteins was significantly upregulated.

Metronidazole resistance in B. fragilis and H. pylori involves genes encoding nitroreductases and oxidoreductases [46,47]. These enzymes activate metronidazole leading to DNA damage and ultimately to cell death [37]. However, changing the abundance of nitroreductases and oxidoreductases might not be the first strategy of $C$. difficile to adapt to metronidazole. Still, the alteration of enzyme activity by adaptation of protein amount, post-translational protein modification or even mutations in their encoding genes, as described earlier for B. fragilis and H. pylori may be viable during long-term adaptation to metronidazole.

In summary, although differences in the species examined and the experimental conditions of the different studies are obvious, the already published effects are in accordance with the data of the current study.

\subsection{Detoxification of Antimicrobial Compounds in C. difficile}

Besides repel, interception, and export, antimicrobial compounds can be detoxified by degradation or modification. During metronidazole stress the synthesis rate of the tellurium resistance protein TerD was found to be increased in C. difficile. It is known from other pathogenic bacteria that these genes mediate responses to diverse extracellular stimuli and may also be involved in detoxification of antimicrobials [48]. However, the mechanisms of detoxification by tellurium resistance proteins are still unknown [49]. In the case of metronidazole stress in C. difficile 630 $\Delta \mathrm{erm}$ the synthesis of two of the five paralogues of TerD were significantly increased suggesting a function of Ter-proteins in Clostridiales during detoxification of metronidazole as well.

During adaptation to antibiotics, the alteration of transcriptional regulators in terms of amount or post-transcriptional modification possibly enables the cell to react to the stress in a swift and comprehensive manner. One of the altered transcriptional regulators, CDIF630erm_01543, was found 
with increased amounts after fidaxomicin stress. This protein can be assigned to the GntR-family, whose members fulfil various biological functions in diverse bacterial groups. In Mycobacterium spec. it is known that GntR-family proteins are involved in resistance to antimicrobial compounds. In detail, overexpression of the GntR-family protein Rv1152 from M. tuberculosis results in higher resistance towards vancomycin [50]. In M. smegmatis overexpression of the GntR-family protein Ms0535 leads to enhanced resistance towards isoniazid, whose metabolite inhibits cell wall synthesis [51]. Additionally, the data obtained in this study may suggest a putative role of GntR-family proteins in the detoxification of the transcriptional inhibitor fidaxomicin.

The other transcriptional regulator altered in C. difficile in response to fidaxomicin stress is the MarR-homologue encoded by CDIF630erm_00601, whose amount was significantly decreased after treatment. In E. coli, MarR is a repressor, which regulates the expression of a regulon (Mar-regulon). The expression of the Mar-regulon confers resistance to organic solvents [52,53], oxidative stress agents [54], disinfectants [55,56], and multiple antibiotics [57,58]. Indeed, mar mutants showed increased susceptibility to inhibitors of gyrase, topoisomerase, transcription, translation, and cell wall synthesis. Besides repression of the mar $A B C$ operon MarR also regulates other targets in E. coli, namely $\operatorname{arc} A B C$, tolC, ompF, zwf, fum $C$, soxS, and inaA [59]. Although there are homologues of those targets in C. difficile, only one, the ArcA-homologue CDIF630erm_01980, could be identified in the dataset presented here. Indeed, the amount of CDIF630erm_01980 increased more than two-fold after fidaxomicin treatment. However, this regulation was not statistically significant. As mutations in E. coli-MarR result in overexpression of ArcAB causing enhanced multidrug-efflux via TolC and therewith resistance to antimicrobials, it is tempting to speculate that the same effects can be observed in C. difficile and that the application of efflux pumps inhibitors in order to improve the activity of antibiotics (as suggested in Reference [60]) would be a treatment option.

\section{Conclusions}

The increasing incidence of $C$. difficile infection and the occurrence and spread of antibiotic-resistant strains emphasize the need for a detailed knowledge on the mode of action of antibiotics against this pathogen. This knowledge is necessary to understand resistance development and to develop new antimicrobial compounds to treat severe and potentially recurrent $C$. difficile infections. One way to contribute to this goal is the compilation of antibiotic signatures. Quantitative data for $425 \mathrm{C}$. difficile proteins obtained in this study point at very distinct proteomic adaptations of this important health-care associated pathogen to the antibiotics $C$. difficile infections are commonly treated with. Moreover, the derived proteomic signatures illustrate that the regulation of protein biosynthesis, DNA replication and repair, as well as the adaptation of the cell envelope are central elements of antibiotics adaptation. The availability of protein signature libraries in C. difficile will not only enhance the knowledge on different mechanisms of action, but will also provide a platform that supports the development and evaluation of new antibiotics.

Supplementary Materials: The following are available online at http://www.mdpi.com/2073-4409/7/11/213/s1, Table S1: All data on protein amounts, Table S2: All data on protein synthesis.

Author Contributions: Conceptualization, S.M. and D.B.; methodology, S.M. and D.A.; experiments, S.M.; data analysis, S.M, A.O. and A.T.-S.; Writing—original draft preparation, S.M.; Writing-review and editing, S.M., A.O., K.R., A.T.-S. and D.B; visualization, S.M. and A.O.; supervision, D.B.; project administration, D.B.; funding acquisition, K.R. and D.B.

Funding: This work was funded by the Federal State of Lower Saxony, Niedersächsisches Vorab (VWZN2889) and the Federal State of Mecklenburg-Pomerania (UG 14 001; UG 16 001). We acknowledge support for the Article Processing Charge from the DFG (German Research Foundation, 393148499) and the Open Access Publication Fund of the University of Greifswald.

Acknowledgments: We acknowledge Ute Kuhrt and Sebastian Grund for excellent technical assistance and Thomas Riedel for help with Proteinortho. Holger Brüggemann and Marie-Laurence Lemay supported this work by critical reading of the manuscript.

Conflicts of Interest: The authors declare no conflict of interest. 


\section{References}

1. Lawson, P.A.; Citron, D.M.; Tyrrell, K.L.; Finegold, S.M. Reclassification of Clostridium difficile as Clostridioides difficile (Hall and O’Toole 1935) Prévot 1938. Anaerobe 2016, 40, 95-99. [CrossRef] [PubMed]

2. Mergenhagen, K.A.; Wojciechowski, A.L.; Paladino, J.A. A review of the economics of treating Clostridium difficile infection. Pharmacoeconomics 2014, 32, 639-650. [CrossRef] [PubMed]

3. Wilcox, M.H.; Howe, R. Diarrhoea caused by Clostridium difficile: Response time for treatment with metronidazole and vancomycin. J. Antimicrob. Chemother. 1995, 36, 673-679. [CrossRef] [PubMed]

4. Gerding, D.N. Treatment of Clostridium difficile-associated diarrhea and colitis. Curr. Top. Microbiol. Immunol. 2000, 250, 127-139. [CrossRef] [PubMed]

5. Surawicz, C.M.; Brandt, L.J.; Binion, D.G.; Ananthakrishnan, A.N.; Curry, S.R.; Gilligan, P.H.; McFarland, L.V.; Mellow, M.; Zuckerbraun, B.S. Guidelines for diagnosis, treatment, and prevention of Clostridium difficile infections. Am. J. Gastroenterol. 2013, 108, 478-498. [CrossRef] [PubMed]

6. Perkins, H.R. Specificity of combination between mucopeptide precursors and vancomycin or ristocetin. Biochem. J. 1969, 111, 195-205. [CrossRef] [PubMed]

7. Gerding, D.N. Is there a relationship between vancomycin-resistant enterococcal infection and Clostridium difficile infection? Clin. Infect. Dis. 1997, 25, S206-S210. [CrossRef] [PubMed]

8. Gajdács, M.; Spengler, G.; Urbán, E. Identification and antimicrobial susceptibility testing of anaerobic bacteria: Rubik's cube of clinical microbiology? Antibiotics 2017, 6, 25. [CrossRef] [PubMed]

9. Miller, W.R.; Murray, B.E.; Rice, L.B.; Arias, C.A. Vancomycin-resistant enterococci: Therapeutic challenges in the 21st century. Infect. Dis. Clin. N. Am. 2016, 30, 415-439. [CrossRef] [PubMed]

10. Barbut, F.; Decré, D.; Burghoffer, B.; Lesage, D.; Delisle, F.; Lalande, V.; Delmée, M.; Avesani, V.; Sano, N.; Coudert, C.; et al. Antimicrobial susceptibilities and serogroups of clinical strains of Clostridium difficile isolated in France in 1991 and 1997. Antimicrob. Agents Chemother. 1999, 43, 2607-2611. [CrossRef] [PubMed]

11. Peláez, T.; Alcalá, L.; Alonso, R.; Rodríguez-Créixems, M.; García-Lechuz, J.M.; Bouza, E. Reassessment of Clostridium difficile susceptibility to metronidazole and vancomycin. Antimicrob. Agents Chemother. 2002, 46, 1647-1650. [CrossRef] [PubMed]

12. Wong, S.S.-Y.; Woo, P.C.-Y.; Luk, W.-K.; Yuen, K.-Y. Susceptibility testing of Clostridium difficile against metronidazole and vancomycin by disk diffusion and Etest. Diagn. Microbiol. Infect. Dis. 1999, 34, 1-6. [CrossRef]

13. Brazier, J.S.; Fawley, W.; Freeman, J.; Wilcox, M.H. Reduced susceptibility of Clostridium difficile to metronidazole. J. Antimicrob. Chemother. 2001, 48, 741-742. [CrossRef] [PubMed]

14. Kuriyama, A.; Jackson, J.L.; Doi, A.; Kamiya, T. Metronidazole-induced central nervous system toxicity: A systematic review. Clin. Neuropharmacol. 2011, 34, 241-247. [CrossRef] [PubMed]

15. Khanna, S.; Pardi, D.S. Clostridium difficile infection: New insights into management. Mayo Clin. Proc. 2012, 87, 1106-1117. [CrossRef] [PubMed]

16. Louie, T.J.; Emery, J.; Krulicki, W.; Byrne, B.; Mah, M. Opt-80 eliminates Clostridium difficile and is sparing of Bacteroides species during treatment of C. difficile infection. Antimicrob. Agents Chemother. 2009, 53, 261-263. [CrossRef] [PubMed]

17. Shue, Y.K.; Sears, P.S.; Shangle, S.; Walsh, R.B.; Lee, C.; Gorbach, S.L.; Okumu, F.; Preston, R.A. Safety, tolerance, and pharmacokinetic studies of Opt-80 in healthy volunteers following single and multiple oral doses. Antimicrob. Agents Chemother. 2008, 52, 1391-1395. [CrossRef] [PubMed]

18. Artsimovitch, I.; Seddon, J.; Sears, P. Fidaxomicin is an inhibitor of the initiation of bacterial RNA synthesis. Clin. Infect. Dis. Off. Publ. Infect. Dis. Soc. Am. 2012, 55 (Suppl. 2), S127-S131. [CrossRef] [PubMed]

19. Cornely, O.A.; Nathwani, D.; Ivanescu, C.; Odufowora-Sita, O.; Retsa, P.; Odeyemi, I.A.O. Clinical efficacy of fidaxomicin compared with vancomycin and metronidazole in Clostridium difficile infections: A meta-analysis and indirect treatment comparison. J. Antimicrob. Chemother. 2014, 69, 2892-2900. [CrossRef] [PubMed]

20. Louie, T.J.; Cannon, K.; Byrne, B.; Emery, J.; Ward, L.; Eyben, M.; Krulicki, W. Fidaxomicin preserves the intestinal microbiome during and after treatment of Clostridium difficile infection (CDI) and reduces both toxin reexpression and recurrence of CDI. Clin. Infect. Dis. Off. Publ. Infect. Dis. Soc. Am. 2012, 55 (Suppl. 2), S132-S142. [CrossRef] [PubMed]

21. Wösten, M.M. Eubacterial sigma-factors. FEMS Microbiol. Rev. 1998, 22, 127-150. [CrossRef] [PubMed] 
22. Freeman, J.; Wilcox, M.H. Antibiotic activity against genotypically distinct and indistinguishable Clostridium difficile isolates. J. Antimicrob. Chemother. 2001, 47, 244-246. [CrossRef] [PubMed]

23. Fuchs, S.; Zühlke, D.; Pané-Farré, J.; Kusch, H.; Wolf, C.; Reiß, S.; Binh, L.T.N.; Albrecht, D.; Riedel, K.; Hecker, M.; et al. Aureolib-A proteome signature library: Towards an understanding of Staphylococcus aureus pathophysiology. PLoS ONE 2013, 8, e70669. [CrossRef] [PubMed]

24. Hecker, M.; Antelmann, H.; Büttner, K.; Bernhardt, J. Gel-based proteomics of Gram-positive bacteria: A powerful tool to address physiological questions. Proteomics 2008, 8, 4958-4975. [CrossRef] [PubMed]

25. Bandow, J.E.; Brötz, H.; Leichert, L.I.O.; Labischinski, H.; Hecker, M. Proteomic approach to understanding antibiotic action. Antimicrob. Agents Chemother. 2003, 47, 948-955. [CrossRef] [PubMed]

26. Hussain, H.A.; Roberts, A.P.; Mullany, P. Generation of an erythromycin-sensitive derivative of Clostridium difficile strain $630(630 \Delta \mathrm{erm})$ and demonstration that the conjugative transposon Tn916 $\mathrm{E}$ enters the genome of this strain at multiple sites. J. Med. Microbiol. 2005, 54, 137-141. [CrossRef] [PubMed]

27. Bernhardt, J.; Weibezahn, J.; Scharf, C.; Hecker, M. Bacillus subtilis during feast and famine: Visualization of the overall regulation of protein synthesis during glucose starvation by proteome analysis. Genome Res. 2003, 13, 224-237. [CrossRef] [PubMed]

28. Bradford, M.M. A rapid and sensitive method for the quantitation of microgram quantities of protein utilizing the principle of protein-dye binding. Anal. Biochem. 1976, 72, 248-254. [CrossRef]

29. Schwarz, K.; Fiedler, T.; Fischer, R.-J.; Bahl, H. A Standard Operating Procedure (SOP) for the preparation of intra- and extracellular proteins of Clostridium acetobutylicum for proteome analysis. J. Microbiol. Methods 2007, 68, 396-402. [CrossRef] [PubMed]

30. Moche, M.; Albrecht, D.; Maaß, S.; Hecker, M.; Westermeier, R.; Büttner, K. The new horizon in 2D electrophoresis-new technology to increase resolution and sensitivity. Electrophoresis 2013, 34, 1510-1518. [CrossRef] [PubMed]

31. Dannheim, H.; Riedel, T.; Neumann-Schaal, M.; Bunk, B.; Schober, I.; Spröer, C.; Chibani, C.M.; Gronow, S.; Liesegang, H.; Overmann, J.; et al. Manual curation and reannotation of the genomes of Clostridium difficile 630 $\mathrm{erm}$ and Clostridium difficile 630. J. Med. Microbiol. 2017, 66, 286-293. [CrossRef] [PubMed]

32. Dembek, M.; Barquist, L.; Boinett, C.J.; Cain, A.K.; Mayho, M.; Lawley, T.D.; Fairweather, N.F.; Fagan, R.P. High-throughput analysis of gene essentiality and sporulation in Clostridium difficile. mBio 2015, 6, e02383-14. [CrossRef] [PubMed]

33. Lechner, M.; Findeiß, S.; Steiner, L.; Marz, M.; Stadler, P.F.; Prohaska, S.J. Proteinortho: Detection of (Co-)orthologs in large-scale analysis. BMC Bioinform. 2011, 12, 124. [CrossRef] [PubMed]

34. Wenzel, M.; Bandow, J.E. Proteomic signatures in antibiotic research. Proteomics 2011, 11, 3256-3268. [CrossRef] [PubMed]

35. Goldstein, E.J.C.; Citron, D.M.; Tyrrell, K.L.; Warren, Y.A. Bactericidal activity of telavancin, vancomycin and metronidazole against Clostridium difficile. Anaerobe 2010, 16, 220-222. [CrossRef] [PubMed]

36. Haft, D.H.; Selengut, J.D.; White, O. The TIGRFAMs database of protein families. Nucleic Acids Res. 2003, 31, 371-373. [CrossRef] [PubMed]

37. Declerck, P.J.; De Ranter, C.J. In vitro reductive activation of nitroimidazoles. Biochem. Pharmacol. 1986, 35, 59-61. [CrossRef]

38. Neumann-Schaal, M.; Hofmann, J.D.; Will, S.E.; Schomburg, D. Time-resolved amino acid uptake of Clostridium difficile 630 $\mathrm{erm}$ and concomitant fermentation product and toxin formation. BMC Microbiol. 2015, 15, 281. [CrossRef] [PubMed]

39. Maaß, S.; Wachlin, G.; Bernhardt, J.; Eymann, C.; Fromion, V.; Riedel, K.; Becher, D.; Hecker, M. Highly precise quantification of protein molecules per cell during stress and starvation responses in Bacillus subtilis. Mol. Cell. Proteomics 2014, 13, 2260-2276. [CrossRef]

40. Hessling, B.; Bonn, F.; Otto, A.; Herbst, F.-A.; Rappen, G.-M.; Bernhardt, J.; Hecker, M.; Becher, D. Global proteome analysis of vancomycin stress in Staphylococcus aureus. Int. J. Med. Microbiol. 2013, 303, 624-634. [CrossRef] [PubMed]

41. VanBogelen, R.A.; Neidhardt, F.C. Ribosomes as sensors of heat and cold shock in Escherichia coli. Proc. Natl. Acad. Sci. USA 1990, 87, 5589-5593. [CrossRef] [PubMed]

42. Chong, P.M.; Lynch, T.; McCorrister, S.; Kibsey, P.; Miller, M.; Gravel, D.; Westmacott, G.R.; Mulvey, M.R. Canadian Nosocomial Infection Surveillance Program (CNISP). Proteomic analysis of a NAP1 Clostridium difficile clinical isolate resistant to metronidazole. PLoS ONE 2014, 9, e82622. [CrossRef] [PubMed] 
43. Emerson, J.E.; Stabler, R.A.; Wren, B.W.; Fairweather, N.F. Microarray analysis of the transcriptional responses of Clostridium difficile to environmental and antibiotic stress. J. Med. Microbiol. 2008, 57, 757-764. [CrossRef] [PubMed]

44. Steffens, L.S.; Nicholson, S.; Paul, L.V.; Nord, C.E.; Patrick, S.; Abratt, V.R. Bacteroides fragilis RecA protein overexpression causes resistance to metronidazole. Res. Microbiol. 2010, 161, 346-354. [CrossRef] [PubMed]

45. Jeong, J.Y.; Mukhopadhyay, A.K.; Dailidiene, D.; Wang, Y.; Velapatiño, B.; Gilman, R.H.; Parkinson, A.J.; Nair, G.B.; Wong, B.C.; Lam, S.K.; et al. Sequential inactivation of $r d x A$ (HP0954) and frxA(HP0642) nitroreductase genes causes moderate and high-level metronidazole resistance in Helicobacter pylori. J. Bacteriol. 2000, 182, 5082-5090. [CrossRef] [PubMed]

46. Gal, M.; Brazier, J.S. Metronidazole resistance in Bacteroides spp. carrying nim genes and the selection of slow-growing metronidazole-resistant mutants. J. Antimicrob. Chemother. 2004, 54, 109-116. [CrossRef] [PubMed]

47. Kwon, D.H.; Kato, M.; El-Zaatari, F.A.; Osato, M.S.; Graham, D.Y. Frame-shift mutations in NAD(P)H flavin oxidoreductase encoding gene ( $f r x A)$ from metronidazole resistant Helicobacter pylori ATCC43504 and its involvement in metronidazole resistance. FEMS Microbiol. Lett. 2000, 188, 197-202. [CrossRef] [PubMed]

48. Taylor, D.E. Bacterial tellurite resistance. Trends Microbiol. 1999, 7, 111-115. [CrossRef]

49. Chasteen, T.G.; Fuentes, D.E.; Tantaleán, J.C.; Vásquez, C.C. Tellurite: History, oxidative stress, and molecular mechanisms of resistance. FEMS Microbiol. Rev. 2009, 33, 820-832. [CrossRef] [PubMed]

50. Zeng, J.; Deng, W.; Yang, W.; Luo, H.; Duan, X.; Xie, L.; Li, P.; Wang, R.; Fu, T.; Abdalla, A.E.; et al. Mycobacterium tuberculosis Rv1152 is a novel GntR family transcriptional regulator involved in intrinsic vancomycin resistance and is a potential vancomycin adjuvant target. Sci. Rep. 2016, 6, 28002. [CrossRef] [PubMed]

51. Hu, J.; Zhao, L.; Yang, M. A GntR family transcription factor positively regulates mycobacterial isoniazid resistance by controlling the expression of a putative permease. BMC Microbiol. 2015, 15, 214. [CrossRef] [PubMed]

52. White, D.G.; Goldman, J.D.; Demple, B.; Levy, S.B. Role of the acrAB locus in organic solvent tolerance mediated by expression of marA, soxS, or robA in Escherichia coli. J. Bacteriol. 1997, 179, 6122-6126. [CrossRef] [PubMed]

53. Aono, R. Improvement of organic solvent tolerance level of Escherichia coli by overexpression of stress-responsive genes. Extremophiles 1998, 2, 239-248. [CrossRef] [PubMed]

54. Ariza, R.R.; Cohen, S.P.; Bachhawat, N.; Levy, S.B.; Demple, B. Repressor mutations in the marRAB operon that activate oxidative stress genes and multiple antibiotic resistance in Escherichia coli. J. Bacteriol. 1994, 176, 143-148. [CrossRef] [PubMed]

55. Moken, M.C.; McMurry, L.M.; Levy, S.B. Selection of multiple-antibiotic-resistant (mar) mutants of Escherichia coli by using the disinfectant pine oil: Roles of the mar and acrAB loci. Antimicrob. Agents Chemother. 1997, 41, 2770-2772. [CrossRef] [PubMed]

56. McMurry, L.M.; Oethinger, M.; Levy, S.B. Overexpression of $\operatorname{mar} A$, soxS, or acr $A B$ produces resistance to triclosan in laboratory and clinical strains of Escherichia coli. FEMS Microbiol. Lett. 1998, 166, 305-309. [CrossRef] [PubMed]

57. Okusu, H.; Ma, D.; Nikaido, H. AcrAB efflux pump plays a major role in the antibiotic resistance phenotype of Escherichia coli multiple-antibiotic-resistance (Mar) mutants. J. Bacteriol. 1996, 178, 306-308. [CrossRef] [PubMed]

58. Goldman, J.D.; White, D.G.; Levy, S.B. Multiple antibiotic resistance (mar) locus protects Escherichia coli from rapid cell killing by fluoroquinolones. Antimicrob. Agents Chemother. 1996, 40, 1266-1269. [CrossRef] [PubMed]

59. Alekshun, M.N.; Levy, S.B. The mar regulon: Multiple resistance to antibiotics and other toxic chemicals. Trends Microbiol. 1999, 7, 410-413. [CrossRef]

60. Spengler, G.; Kincses, A.; Gajdács, M.; Amaral, L. New roads leading to old destinations: Efflux pumps as targets to reverse multidrug resistance in bacteria. Molecules 2017, 22, 468. [CrossRef] [PubMed]

(C) 2018 by the authors. Licensee MDPI, Basel, Switzerland. This article is an open access article distributed under the terms and conditions of the Creative Commons Attribution (CC BY) license (http:/ / creativecommons.org/licenses/by/4.0/). 